\title{
Finite-size scaling at first-order quantum transitions
}

\author{
Massimo Campostrini, ${ }^{1}$ Jacopo Nespolo, ${ }^{1}$ Andrea Pelissetto, ${ }^{2}$ and Ettore Vicari ${ }^{1}$ \\ 1 Dipartimento di Fisica dell'Università di Pisa and INFN, Largo Pontecorvo 3, I-56127 Pisa, Italy and \\ ${ }^{2}$ Dipartimento di Fisica di "Sapienza," Università di Roma and INFN, Sezione di Roma I, I-00185 Roma, Italy
}

(Dated: June 24, 2021)

\begin{abstract}
We study finite-size effects at first-order quantum transitions (FOQTs). We show that the lowenergy properties show a finite-size scaling (FSS) behavior, the relevant scaling variable being the ratio of the energy associated with the perturbation driving the transition and the finite-size energy gap at the FOQT point. The size dependence of the scaling variable is therefore essentially determined by the size dependence of the gap at the transition, which in turn depends on the boundary conditions. Our results have broad validity and, in particular, apply to any FOQT characterized by the degeneracy and crossing of the two lowest-energy states in the infinite-volume limit. In this case, a phenomenological two-level theory provides exact expressions for the scaling functions. $\mathrm{Nu}-$ merical results for the quantum Ising chain in transverse and parallel magnetic fields support the FSS ansatzes.
\end{abstract}

PACS numbers: 05.30.Rt,64.60.an,05.70.Fh

Zero-temperature quantum phase transitions (QPT) are phenomena of great interest in different branches of physics [1, 2]. They arise in many-body systems with competing ground states controlled by nonthermal parameters. QPT are continuous when the ground state of the system changes continuously at the transition point and correlation functions show a divergent length scale. They are instead of first order when the ground-state properties are discontinuous across the transition point.

These singular behaviors are observed in the infinitevolume limit. If the size $L$ of the system is finite, all properties are generally analytic as a function of the external parameter $\mu$ driving the transition. However, around the transition point, low-energy thermodynamic quantities and large-scale structural properties show a finite-size scaling (FSS) behavior depending on the nature and on the general properties of the transition. An understanding of these finite-size properties is important for a correct interpretation of experimental or numerical data when phase transitions are investigated in relatively

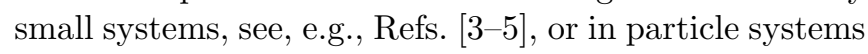
trapped by external forces, as in cold-atom experiments, see, e.g., Ref. [6].

At continuous transitions a FSS behavior is observed when the length scale $\xi$ of the critical modes becomes comparable with the size $L$ of the system. For large values of $L$, this regime presents universal features, shared by all systems whose transition belongs to the same universality class. Although originally formulated in the classical framework [7], FSS extends to quantum continuous transitions exploiting the quantum-to-classical mapping [8, 9].

First-order quantum transitions (FOQTs) are also of great interest, as they occur in a large number of quantum many-body systems, such as quantum Hall samples 10], itinerant ferromagnets [1], heavy-fermion metals 12 14], etc. FOQTs occur for those values $\mu_{c}$ of the external driving parameter $\mu$ at which the lowest energy states show a level crossing. Since the ground state is different for $\mu<\mu_{c}$ and $\mu>\mu_{c}$, the physical properties change discontinuously at $\mu_{c}$. In the absence of particular conservation laws, a level crossing can only occur in the infinite-volume limit. In a finite system, the presence of nonvanishing matrix elements among these states lifts the degeneracy, giving rise to the phenomenon of avoided level crossing. In this paper we investigate this issue, in particular we study the interplay between the size $L$ of the system and the parameter $\mu$ driving the transition.

First-order classical transitions (FOCTs), driven by thermal fluctuations, show FSS behaviors $\underline{3}, 15$-19 that are somewhat analogous to those observed at continuous transitions. On the basis of the general quantumto-classical mapping of $d$-dimensional quantum systems onto classical anisotropic $(d+1)$-dimensional systems, we expect FSS also at FOQTs. Here we will show that this is indeed the case, identifying the general scaling variables which parametrize FSS.

To make the discussion concrete, we start by considering a generic finite $d$-dimensional cubic system of size $L$ which undergoes a FOQT driven by a magnetic field $h$. We wish to determine the scaling behavior of the energy differences of the lowest states $\Delta_{n}(L, h)=$ $E_{n}(L, h)-E_{0}(L, h)$ and of the magnetization $m(L, h)$. On dimensional grounds, we expect that around $h=0$ the relevant scaling variable is the ratio between the energy contribution of the magnetic field and the gap $\Delta_{L} \equiv \Delta_{1}(L, h=0)$ at $h=0$, i.e.,

$$
\kappa \sim h L^{d} / \Delta_{L} .
$$

Then, if a scaling behavior is realized, the energy difference of the two lowest energy states for finite $h$ should satisfy the scaling ansatz

$$
\Delta(L, h) \equiv \Delta_{1}(L, h) \approx \Delta_{L} f_{\Delta}(\kappa) .
$$

We have $f_{\Delta}(0)=1$ by definition, and $f_{\Delta}(\kappa) \sim|\kappa|$ for $\kappa \rightarrow \pm \infty$ in order to reproduce the expected linear be- 
havior $\Delta(L, h) \sim|h| L^{d}$ for sufficiently large $|h|$. The magnetization is expected to scale as

$$
m(L, h) \approx m_{0} f_{\sigma}(\kappa),
$$

where $m_{0}$ is the magnetization obtained approaching the transition point $h \rightarrow 0$ after the infinite-volume limit. Because of the definition of $m_{0}$, we have $f_{\sigma}(\kappa) \rightarrow \pm 1$ for $\kappa \rightarrow \pm \infty$. Moreover, $f_{\sigma}(0)=0$ due to the parity symmetry when $h=0$. The above FSS ansatzes represent the simplest scaling behaviors compatible with the discontinuities arising in the infinite-volume limit. We can also extend the scaling ansatz to include the temperature $T$. In the scaling limit $T \rightarrow 0$, similar dimensional arguments indicate that the relevant scaling variable is the ratio $\rho=T / \Delta_{L}$, so that, at finite small $T$ we predict, for instance,

$$
m(L, h, T) \approx m_{0} F_{\sigma}(\kappa, \rho) .
$$

The scaling variables $\kappa$ and $\rho$ are related to the finitesize energy gap at the transition, whose size behavior depends crucially on the boundary conditions considered. Hence, once they are expressed in terms of the parameter driving the transition and the size $L$, their $L$ dependence changes according to the chosen boundary conditions. As we shall show, it may be proportional to powers of $L$ as in continuous phase transitions, or be proportional to exponentials of $L$, as it occurs in Ising classical systems with cylindrical geometry [17].

Let us now additionally assume that the transition is due to the crossing of the two lowest-energy states in the infinite-volume limit. The higher excited states are instead assumed to be gapped in the same limit. For example, this occurs in the quantum ferromagnetic phase of Ising-like systems with appropriate boundary conditions, see below. In this case, Eqs. (2) and (3) can be obtained by a phenomenological theory, which additionally provides the expressions of the scaling functions. Because of the hypotheses made, for sufficiently small $|h|$ the difference between the energies of the lowest states is much smaller than those between the higher excited states and the ground state, i.e.

$$
\Delta(L, h) \equiv \Delta_{1}(L, h) \ll \Delta_{n}(L, h) \text { for } n>1 .
$$

Thus, it is natural to assume that the low-energy properties in the crossover region around $h=0$ are simply obtained by restricting the theory to the two lowest-energy states. The Hamiltonian restricted to this subspace has the general form

$$
H_{r}=\left(\begin{array}{ll}
\varepsilon+\beta & \delta e^{i \varphi} \\
\delta e^{-i \varphi} & \varepsilon-\beta
\end{array}\right)
$$

where the basis is chosen so that $\beta \sigma^{(3)}$ represents the perturbation induced by the magnetic field $h\left(\sigma^{(3)}\right.$ is the diagonal Pauli matrix), thus $\beta \approx m_{0} h L^{d}$. $\delta$ is a small parameter which vanishes for $L \rightarrow \infty$ and $h=0$, in order to obtain a degenerate ground state. Diagonalizing $H_{r}$, we obtain the energy difference of the two eigenstates:

$$
E_{1}-E_{0}=2 \delta \sqrt{1+(\beta / \delta)^{2}} .
$$

This expression is consistent with the scaling ansatz (2), provided that we identify

$$
2 \delta \rightarrow \Delta_{L}, \quad \kappa \equiv \frac{\beta}{\delta} \rightarrow \frac{2 m_{0} h L^{d}}{\Delta_{L}},
$$

and

$$
f_{\Delta}(\kappa)=\sqrt{1+\kappa^{2}} .
$$

Moreover, by computing the expectation value of $\sigma^{(3)}$ on the lowest eigenstate, we obtain that the magnetization satisfies the scaling relation (3), with

$$
f_{\sigma}(\kappa)=\frac{\kappa^{2}+\kappa \sqrt{1+\kappa^{2}}}{1+\kappa^{2}+\kappa \sqrt{1+\kappa^{2}}} .
$$

The FSS functions (9) and (10) parametrize $\Delta(L, h)$ and $m(L, h)$ in the limit $L \rightarrow \infty$ keeping $\kappa$ fixed. In this limit, scaling corrections are expected to be suppressed by powers of the inverse size. Note that the same technique also provides a framework to study the unitary quantum dynamics when $h$ varies (in time) in a small interval around $h=0$. Since only the two lowest-energy states are relevant, the dynamics is analogous to that governing a two-level quantum mechanical system in which the energy separation of the two levels is a function of time (the Landau-Zener effect [20]).

In principle, the scaling ansatzes can be derived by exploiting the general quantum-to-classical mapping, which allows us to map the quantum system onto a classical one defined in an anisotropic box of volume $V=L^{d} L_{t}$, with $L_{t} \sim 1 / T$ and $L_{t} \gg L$. We can therefore check our general predictions using the results of Ref. [17] for Ising-like systems with PBC along the transverse dimensions. In the classical case, FSS is characterized by the scaling variables $u \sim h V$ and $v \sim \xi_{t} / L_{t}$, where $\xi_{t}$ is the characteristic length at the transition: $\xi_{t} \sim \exp \left(L^{d} \sigma\right)$, where $\sigma$ is the interfacial tension. These results are fully consistent with our ansatz. If we identify $\Delta_{L}$ with $1 / \xi_{t}$, we obtain the correspondence $u / v \rightarrow \kappa$ and $v \rightarrow \rho$ between the variables $\kappa \sim h L^{d} / \Delta_{L}$ and $\rho \sim T / \Delta_{L}$ and the classical ones $u$ and $v$. Note also that the transfer-matrix analysis of Ref. [17] is analogous to the one presented here, as they also observe an avoided-crossing phenomenon.

To verify the general predictions, we consider a quantum Ising chain of size $L$ in a transverse and parallel magnetic field. Its Hamiltonian is

$$
H_{\mathrm{Is}}=-J \sum_{i} \sigma_{i}^{(1)} \sigma_{i+1}^{(1)}-g \sum_{i} \sigma_{i}^{(3)}-h \sum_{i} \sigma_{i}^{(1)},
$$



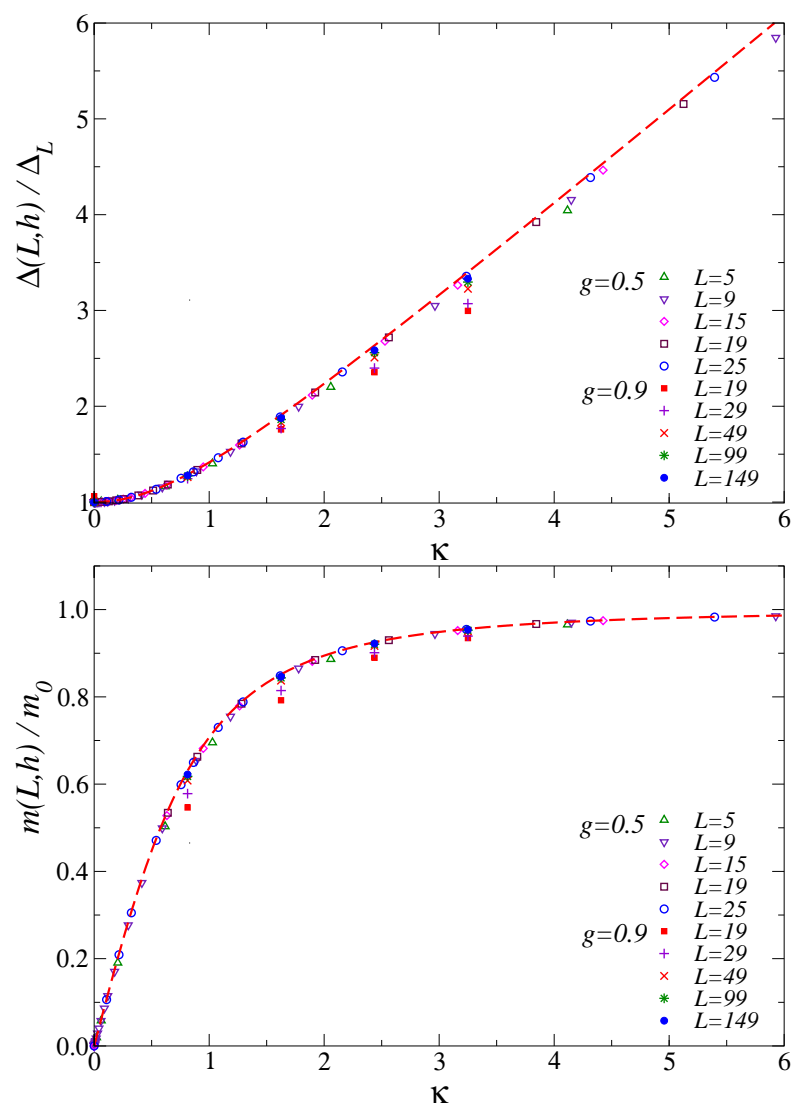

FIG. 1: (Color online) FSS of the energy difference $\Delta(L, h)$ of the lowest states and of the central-site magnetization $m(L, h)$ for the Ising chain with OBC, at $g=0.5$ and $g=0.9$. We plot the ratios $\Delta(L, h) / \Delta_{L}$ (top) and $m(L, h) / m_{0}$ (bottom) versus $\kappa=2 m_{0} h L / \Delta_{L}$. We use $m_{0}=\left(1-g^{2}\right)^{1 / 8}$ and approximate $\Delta_{L}$ with its leading term, i.e., we set $\Delta_{L}=2\left(1-g^{2}\right) g^{L}$ (corrections are small, of order $\left.g^{2 L}\right)$. The dashed lines correspond to the scaling functions (9) and (10).

where $\sigma_{i}^{(a)}$ are the Pauli matrices. For $h=0$ model (11) has a continuous transition at $g=1$ (we set $J=1$ and assume $g>0$ ), belonging to the $2 D$ Ising universality class. This quantum critical point separates a paramagnetic $(g>1)$ and a ferromagnetic $(g<1)$ phase. In the ferromagnetic phase, the quantum Ising chain shows a FOQT at $h=0$ for any $g<1$, with a discontinuity of the magnetization, i.e. the ground-state expectation value of $\sigma_{i}^{(1)}$. The Ising chain is an ideal theoretical laboratory, because several exact results are known for $h=0$. In the case of open or periodic boundary conditions (OBC and $\mathrm{PBC}$, respectively), the transition is due to a crossing of the two lowest-energy states $|+\rangle$ and $|-\rangle$, such that

$$
\left\langle \pm\left|\sigma_{i}^{(1)}\right| \pm\right\rangle= \pm m_{0}
$$

(this is strictly true for PBC, in the case of OBC boundary effects are neglected), where 21]

$$
m_{0}=\lim _{h \rightarrow 0^{+}} \lim _{L \rightarrow \infty}\left\langle\sigma_{x}^{(1)}\right\rangle=\left(1-g^{2}\right)^{1 / 8} .
$$

However, in a finite system of size $L$, due to tunneling effects, the lowest states are superpositions of the states $|+\rangle$ and $|-\rangle$. Their energy difference $\Delta_{L}$ vanishes exponentially as $L$ increases: 21, 22]

$$
\Delta_{L} \equiv \Delta_{1}(L, h=0)=2\left(1-g^{2}\right) g^{L}\left[1+O\left(g^{2 L}\right)\right]
$$

in the OBC case, and $\Delta_{L} \approx 2\left[\left(1-g^{2}\right) /(\pi L)\right]^{1 / 2} g^{L}$ in the PBC case. On the other hand, the difference $\Delta_{i}$ for the higher excited states $(i>1)$ is finite for $L \rightarrow \infty$ [23].

Using the density-matrix renormalization-group (DMRG) method 24, 25], we compute the energy difference $\Delta_{1}(L, h)$ of the lowest two states and the ground-state magnetization of Ising chains of odd size $L$, such that $-L / 2 \leq i \leq L / 2$, with OBC. In the case of $\mathrm{OBC}$ there is no translation invariance, thus $M_{i}=\left\langle\sigma_{i}^{(1)}\right\rangle$ depends on $i$. Such a dependence is weak far from the boundary. In the following, we consider $m(L, h) \equiv M_{0}(L, h)$ at the center of the lattice. Fig. 1 shows data at $g=0.5$ and $g=0.9$. They clearly show the predicted FSS behavior. Indeed, the ratios $\Delta(L, h) / \Delta_{L}$ and $m(L, h) / m_{0}$ approach universal, $g$ independent, curves when they are plotted versus $\kappa=2 m_{0} h L / \Delta_{L}$. Moreover, the scaling functions agree with those given by the phenomenological two-level theory, i.e., with Eqs. (9) and (10). These behaviors appear to set in for quite small lattice sizes, with corrections that turn out to asymptotically decrease as $O(1 / L)$.

As an additional check of the general argument, we consider systems in which the parallel magnetic field $h_{j}$ is nonvanishing only at one lattice site $j$, for example at the center of the chain. The general arguments should apply to this case as well, with $\beta \sim h_{j}$ instead of $\beta \sim h L$. Therefore, we expect a FSS behavior analogous to that valid for the homogenous parallel field, the corresponding scaling variable being $\kappa=2 m_{0} h_{j} / \Delta_{L}$. DMRG results for the Ising chain are in full agreement with these scaling predictions. In particular, the scaling behavior of $\Delta(L, h)$ given by Eqs. (2) and (9) can be analytically derived for magnetic fields localized at the boundaries. These calculations will be reported elsewhere.

Although our discussion has essentially focused on Ising-like systems, the two-level theory is general and applies to any FOQT in which two levels cross in the infinite-volume limit. Indeed, the parity symmetry $h \rightarrow$ $-h$ plays no role here, as it can be easily checked by allowing for generic diagonal terms in the matrix (6), i.e. by replacing $\epsilon \pm \beta$ with $\epsilon+\beta_{1}$ and $\epsilon+\beta_{2}$, where $\beta_{1}, \beta_{2}$ represent the energy contributions of the driving perturbation. Moreover, it can be easily extended to systems with a larger finite degeneracy at the transition, keeping into account a larger number of low-energy states.

Finally, we show that the FSS Eqs. (2) and (3) also hold in systems with an infinite degeneracy of the ground state at the transition. To investigate this case, we consider the Ising chain with antiperiodic boundary conditions $(\mathrm{ABC})$, or fixed opposite boundary conditions 


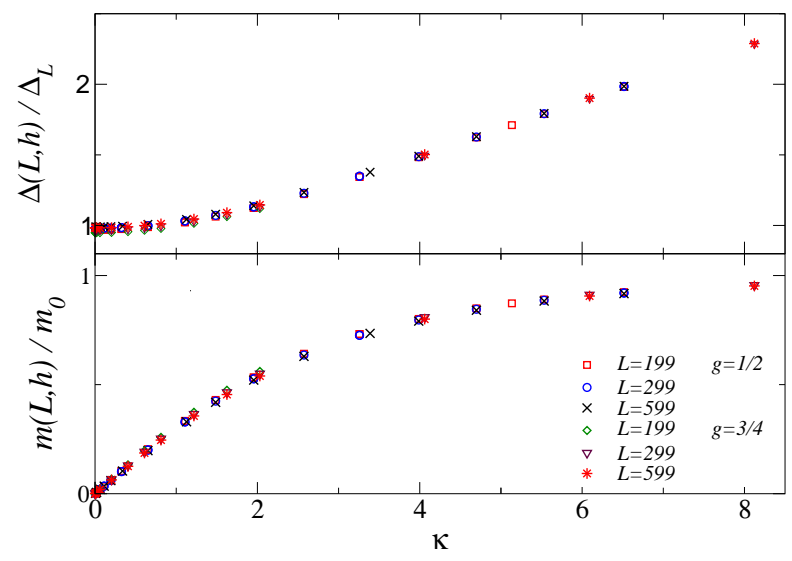

FIG. 2: (Color online) FSS of the energy difference of the lowest states and magnetization of the Ising chain with FOBC, at $g=1 / 2$ and $g=3 / 4$. We plot the ratios $\Delta(L, h) / \Delta_{L}$ (top) and $m(L, h) / m_{0}$ (bottom) versus $\kappa \equiv 2 m_{0} h L / \Delta_{L}$, with $m_{0}$ and $\Delta_{L}$ given by Eqs. (13) and (15). The data approach nontrivial scaling curves with increasing $L$, which are independent of $g$, supporting the FSS Eqs. (2) and (3).

(FOBC) [26]. In these two cases, the lowest-energy states are associated with domain walls (kinks), i.e., with nearest neighbors pairs of antiparallel spins, which can be considered as one-particle states with $O\left(L^{-1}\right)$ momenta. Hence, there is an infinite number of excitations with a gap of order $L^{-2}$. In particular, we have

$$
\Delta_{L} \equiv \Delta_{1}(L, h=0)=c \frac{g}{1-g} \frac{\pi^{2}}{L^{2}}+O\left(L^{-3}\right)
$$

with $c=1$ for ABC [22] and $c=3$ for FOBC. DMRG calculations show that scaling ansatzes (2) and (3) hold also in this case. Indeed, like the OBC case, the data for the ratios $\Delta(L, h) / \Delta_{L}$ and $m(L, h) / m_{0}$ approach universal (independent of $g$ ) scaling curves when plotted versus $\kappa \equiv 2 m_{0} h L / \Delta_{L}$, see Fig. 2.

Note that, if we express the scaling variable $\kappa$ in terms of $L$, the $L$ dependence is quite different from that observed in the $\mathrm{OBC}$ and $\mathrm{PBC}$ case. While $\kappa \sim h L g^{-L}$ and $\kappa \sim h L^{3 / 2} g^{-L}$ for $\mathrm{OBC}$ and PBC, respectively, we have $\kappa \sim h L^{3}$ for $\mathrm{ABC}$ and FOBC, since $\Delta_{L} \sim L^{-2}$, cf. Eq. (15). Including the temperature in the case of $\mathrm{ABC}$ and FOBC, we obtain the scaling variable $\rho=T L^{2}$, which shows that the FSS limit in the corresponding classical system is strongly anisotropic (the same occurs in the PBC case, already considered in Ref. [17]). These results show that the FSS dependence may significantly depend on the boundary conditions, being strictly connected with the large-volume low-energy structure of the eigenstates.

In conclusion, quantum systems show a universal FSS behavior at FOQTs. For instance, the gap satisfies Eq. (2) when the scaling variable $\kappa$ is expressed in terms of the ratio of the energy associated with the perturbation driving the transition and the finite-size energy gap at the FOQT. We show that these results hold for general FOQTs characterized by the degeneracy and crossing of the two lowest-energy states in the infinite-volume limit, and that, in this case, it is possible to predict the scaling functions. We also show that the same FSS predictions also hold in some systems in which the degeneracy of the ground state at the transition is infinite (this is the case of the Ising chain with $\mathrm{ABC}$ or $\mathrm{FOBC}$ ), indicating that Eq. (2) has a broad range of validity. It is important to note that $\kappa$ depends on $\Delta_{L}$, whose $L$ dependence, in turn, varies with the boundary conditions. This implies that $\kappa$ may show different $L$ dependences for different boundary conditions. For instance, for the Ising chain $\kappa \sim h L e^{\alpha L}$ for $\mathrm{OBC}, \kappa \sim h L^{3 / 2} e^{\alpha L}$ for $\operatorname{PBC}(\alpha$ is a nonuniversal positive constant), and $\kappa \sim h L^{3}$ for $\mathrm{ABC}$ or FOBC. Further work is needed to investigate systems with vector symmetries, which, in the classical case [18], have a more complex behavior due to the presence of massless Goldstone bosons.

The FSS theory at FOQTs should be particularly useful in those cases in which the nature of the transition is not known a priori and only data for relatively small systems are available: FSS may be exploited to discriminate continuous from weak FOQTs. We also note that, in some cases, FSS behaviors are already observed for relatively small systems, for example in the case of Ising chains with OBC, see Fig. 11. Thus, even small systems may show definite signatures of FOQTs, as also argued in Refs. 27, 28]. This makes FSS at FOQTs particularly interesting also experimentally. In particular, quantum simulators 29], in which an extremely high degree of control is achieved in simple systems of a few atoms, have already been used to investigate the quantum critical behavior of different models [28, 30, 31], and could provide experimental support to the scaling theory introduced in the present work.

[1] S. Sachdev, Quantum Phase Transitions, (Cambridge University Press. 2011, 2nd ed.)

[2] M. Vojta, Rep. Prog. Phys. 66, 2069 (2003).

[3] V. Privman ed., Finite Size Scaling and Numerical Simulation of Statistical Systems (World Scientific, Singapore, 1990).

[4] A. Pelissetto and E. Vicari, Phys. Rep. 368, 549 (2002).

[5] F.M. Gasparini, M.O. Kimball, K.P. Mooney, and M. Diaz-Avilla, Rev. Mod. Phys. 80, 1009 (2008).

[6] I. Bloch, J. Dalibard, and W. Zwerger, Rev. Mod. Phys. 80, 885 (2008).

[7] M.E. Fisher and M.N. Barber, Phys. Rev. Lett. 28, 1516 (1972).

[8] S.L. Sondhi, S.M. Girvin, J.P. Carini, and D. Shahar, Rev. Mod. Phys. 69, 315 (1997).

[9] M. Campostrini, A. Pelissetto and E. Vicari, Phys. Rev. B 89, 094516 (2014).

[10] V. Piazza, V. Pellegrini, F. Beltram, W. Wegscheider, 
T. Jungwirth, and A.H. MacDonald, Nature 402, 638 (1999).

[11] T. Vojta, D. Belitz, T.R. Kirkpatrick, and R. Narayanan, Ann. Phys. (Leipzig) 8, 593 (1999).

[12] M. Uhlarz, C. Pfleiderer, and S.M. Hayden, Phys. Rev. Lett. 93, 256404 (2004).

[13] C. Pfleiderer, J. Phys.: Cond. Matter 17, S987 (2005).

[14] W. Knafo, S. Raymond, P. Lejay, and J. Flouquet, Nature Phys. 5, 753 (2009).

[15] B. Nienhuis and M. Nauenberg, Phys. Rev. Lett. 35, 477 (1975).

[16] M.E. Fisher and A.N. Berker, Phys. Rev. B 26, 2507 (1982);

[17] V. Privman and M. E. Fisher, J. Stat. Phys. 33, 385 (1983).

[18] M. E. Fisher and V. Privman, Phys. Rev. B 32, 447 (1985).

[19] M.S.S. Challa, D.P. Landau, and K. Binder, Phys. Rev. B 34, 1841 (1986); K. Vollmayr, J.D. Reger, M. Scheucher, and K. Binder, Z. Phys. B 91, 113 (1993).

[20] C. Zener, Proc. R. Soc. London, Ser A 137, 696 (1932);

L. Landau, Phys. Z. Sowjetunion 2, 46 (1932).

[21] P. Pfeuty, Ann. Phys. 57, 79 (1970).

[22] G.G. Cabrera and R. Jullien, Phys. Rev. B 35, 7062 (1987).
[23] For instance, $\Delta_{2}(L, h=0)=2(1-g)+O\left(L^{-2}\right)$ for OBC and $\Delta_{2}(L, h=0)=4(1-g)+O\left(L^{-2}\right)$ for PBC, respectively.

[24] U. Schollwöck, Rev. Mod. Phys. 77, 259 (2005).

[25] Since the system is always far from criticality, DMRG is extremely efficient; however, for very small energy differences, computation of eigenvectors and therefore of vacuum expectation values can be inaccurate (this is true of any numerical diagonalization method). The precision on the observables is typically $10^{-15}\left|E_{0}\right| / \Delta_{L}$ with $\left|E_{0}\right| \simeq L$.

[26] FOBCs correspond to OBC and an additional boundary term given by $H_{b}=J\left(\sigma_{-L / 2}^{(1)}-\sigma_{L / 2}^{(1)}\right)$.

[27] F. Iachello and N. V. Zamfir, Phys. Rev. Lett. 92, 212501 (2004).

[28] G.-D. Lin, C. Monroe, and L.-M. Duan, Phys. Rev. Lett. 106, 230402 (2011).

[29] I.M. Georgescu, S. Ashhab, and F. Nori, Rev. Mod. Phys. 86, 153 (2014).

[30] R. Islam, E.E. Edwards, K. Kim, S. Korenblit, C. Noh, H. Carmichael, G.-D. Lin, L.-M. Duan, C.-C. Joseph Wang, J.K. Freericks, and C. Monroe, Nature Comm. 2377 (2011).

[31] J. Simon, W.S. Bakr, R. Ma, M.E. Tai, P.M. Preiss, and M. Greiner, Nature 472, 307 (2011). 